\title{
Influence of Autotransformer with Small Ground Reactance to Fault Current
}

\author{
Rong Zhou ${ }^{1}$, Yuqin Yao ${ }^{1, a}$ \\ ${ }^{1}$ Chengdu University of Information Technology, Chengdu, 610225, China \\ ${ }^{a}$ email: yyq@cuit.edu.cn
}

Key Words: autotransformer; small reactance earth; short circuit current

\begin{abstract}
It is an efficient method to improve short circuit impedance that autotransformer is grounded by small reactance. In this paper, the restraining method is analyzed. Firstly, base on zero sequence circuit of autotransformer, it was deduced that the equivalent reactance is increased in this method. Then single phase and three phase fault current in $220 \mathrm{kV}$ side were calculated after connecting $16 \Omega$ reactance. Earth reactance influence to fault current was analyzed with the above results.
\end{abstract}

\section{Overview}

Currently, the power grid is expanded and the voltage level is improved. For the advantages of small volume, low cost, convenient transportation and etc., the $500 / 220 \mathrm{kV}$ autotransformer has been widely used in the power system. The capacity of modern power system became larger and the communication among district nets became closer. Load density is larger and manufacturing technique is added. Hence, zero sequence reactance of autotransformer is small to zero. At this time, the neutral point directly grounded in the $220 \mathrm{kV}$ side autotransformer fault, its single-phase short-circuit current will likely be close to or even exceed the three-phase short-circuit current ${ }^{[1]}$. It is difficult for device selection in $220 \mathrm{kV}$ breaker side and ground net design.

For solving the above problem, increasing short circuit impedance obviously decreases the fault current. However, the reactive power increases. And it also improves the difficulty of transformer manufacturing. If autotransformer uses this way, it will be efficient.

\section{Zero Sequence Impedance of Neutral Point with Small Reactance Earth}

If the neutral grounding via low reactance, then for the general transformer windings when the zero sequence current is passed, there will be a triple zero sequence current flowing through the resistor to ground, and generates a corresponding voltage drop, so that the neutral point potential higher than zero potential earth ${ }^{[2]}$. At this point, the zero sequence impedance is equivalent leakage reactance of the windings and three times the neutral point and small reactance.

For auto-transformers, there is a common coil for its high and medium sides. And they share the same neutral point, and have circuit connection. Among sex via low impedance grounding, the grounding impedance high pressure sides also shared. At this time, the current flowing through the neutral point is three times the difference between the zero-sequence current high pressure side, and the potential of the neutral point equally affected both sides of the zero-sequence current, unlike ordinary transformer ${ }^{[3]}$. The following analysis is about the how earth reactance affects the auto-transformer parameters.

Figure 1 covers the auto-transformer zero sequence circuit in both cases, which are the third winding open or closed. 1,2,3 represent high, medium and low voltage three sides, the neutral point directly to ground (ie, $\mathrm{x}_{\mathrm{n}}=0$ ) when the three sides of the zero-sequence reactance, respectively $\mathrm{x}_{1}, \mathrm{x}_{2}$, $\mathrm{x}_{3}$; when neutral point grounded small reactance $\mathrm{x}_{\mathrm{n}}$ is grounded, three sides of the zero-sequence reactance are respectively $\mathrm{x}_{1}{ }^{\prime}, \mathrm{x}_{2}{ }^{\prime}, \mathrm{x}_{3}{ }^{\prime}$. $\mathrm{U}_{\mathrm{n}}$ is neutral point voltage, $\mathrm{U}_{10}$ and $\mathrm{U}_{20}$ are voltages of high and medium windings. $U_{1 n}$ and $U_{2 n}$ are voltage between winding and neutral point. It could be obtained as follows. 

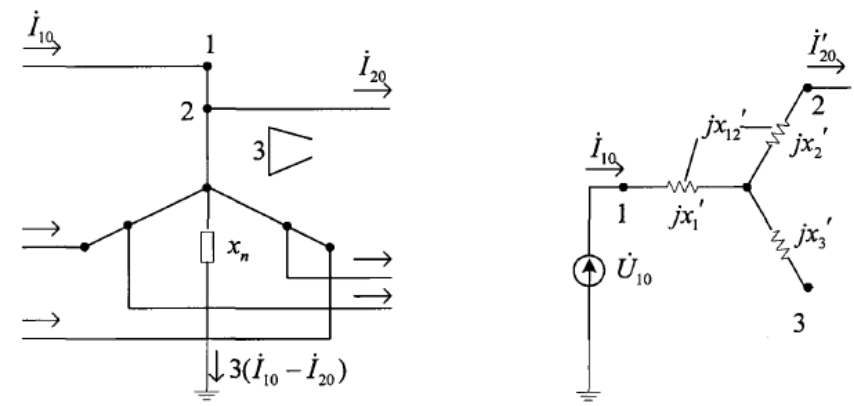

(a) Low pressure side of the opening triangle
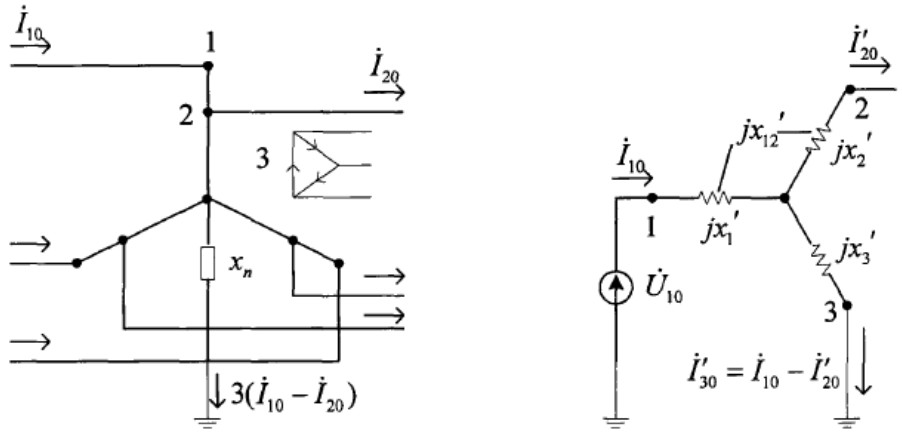

(b) Low side of closed triangle

Fig.1 zero sequence equivalent circuit of auto transformer grounded with small reactance

$$
\left\{\begin{array}{l}
\dot{U}_{10}=\dot{U}_{1 n}+\dot{U}_{n} \\
\dot{U}_{20}=\dot{U}_{2 n}+\dot{U}_{n}
\end{array}\right.
$$

Setting ratio of high and medium side as $\mathrm{k}=\mathrm{U}_{1} / \mathrm{U}_{2}$, the zero sequence equivalent reactance is:

$$
j x_{1}^{\prime}+j x_{2}^{\prime}=j x_{12}^{\prime}=\frac{\dot{U}_{10}-k \dot{U}_{20}}{\dot{I}_{10}}=\frac{\dot{U}_{1 n}-k \dot{U}_{2 n}}{\dot{I}_{10}}+\frac{(1-k) \dot{U}_{n}}{\dot{I}_{10}}
$$

When neutral point is grounded directly, the high side equivalent reactance is:

$$
j x_{12}^{\prime}=\frac{\dot{U}_{1 n}-k \dot{U}_{2 n}}{\dot{I}_{10}}
$$

From Fig.1, the neutral point voltage has the relationship as follows:

$$
\dot{U}_{n}=j 3 x_{n}\left(\dot{I}_{10}-\dot{I}_{20}\right)=j 3 x_{n}(1-k) \dot{I}_{10}
$$

Finishing (2) - (4), it can be obtained by small electric transformer neutral point when anti-grounded, converted to the high-pressure side of the high pressure equivalent zero-sequence reactance:

$$
j x_{12}^{\prime}=j x_{12}+j 3 x_{n}(1-k)^{2}=j x_{1}+j x_{2}+j 3 x_{n}(1-k)^{2}
$$

As can be seen from the formula (5), the increase in this case high pressure compared with the equivalent reactance of the neutral point earthed, and the reactance increases with the ground, which increases the larger the value. This is neutral ground by small electric short circuit current decreases when the anti main reason.

\section{Simulation of Autotransformer with Small Reactance Earthing in Neutral Point}

According to People's Republic of China Electric Power Industry Standard DL/T620-1997 "AC overvoltage protection and insulation of electrical installations" relevant provisions, when the neutral point reactor grounded, its reactance transformer or high voltage shunt reactors zero sequence reactance must be less than $1 / 3^{[4]}$. For maximum single-phase ground short circuit current 
limiting, access the main transformer neutral point reactance transformer can also be greater than zero sequence reactance of $1 / 3$, but the system needs to meet the zero-sequence reactance than the positive sequence reactance of less than 3 this limitation ${ }^{[5]}$. In this Example, the size of the reactance added $16 \Omega$.

In different situation of neutral point without reactance and with $16 \Omega$ reactance, the three phase and single phase fault current are shown as Fig.2 and Fig.3.

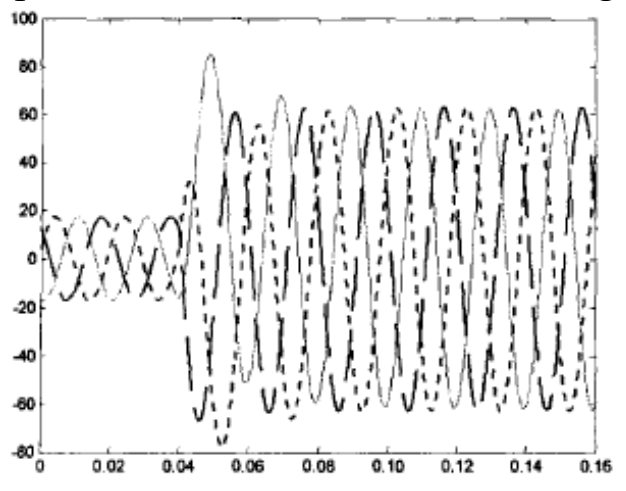

(a) neutral point without reactance

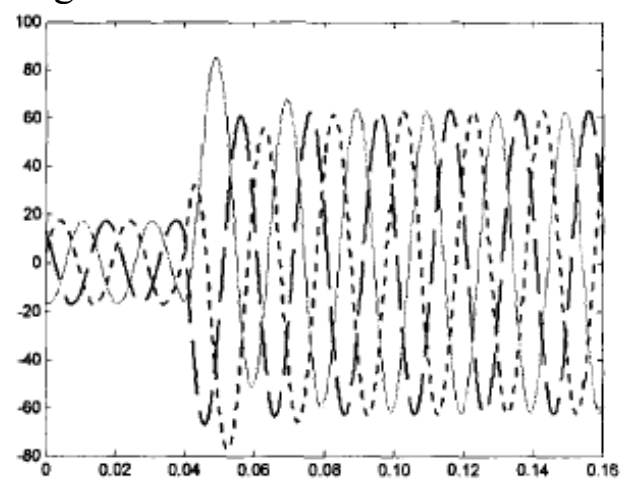

(b) neutral point with $16 \Omega$ reactance

Fig. 2 three phase fault current in $220 \mathrm{kV}$ side

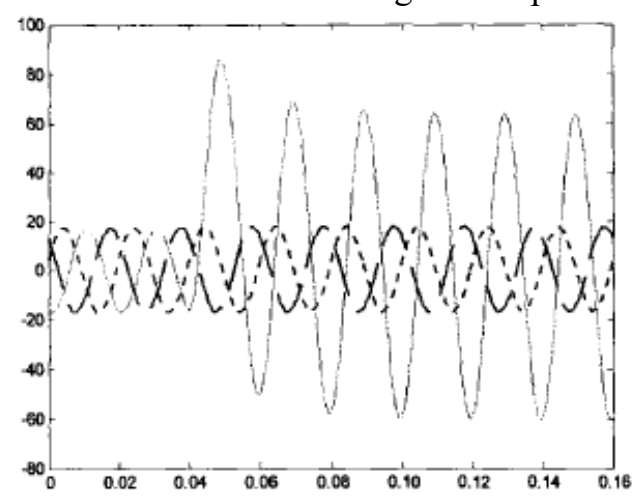

(a) neutral point without reactance

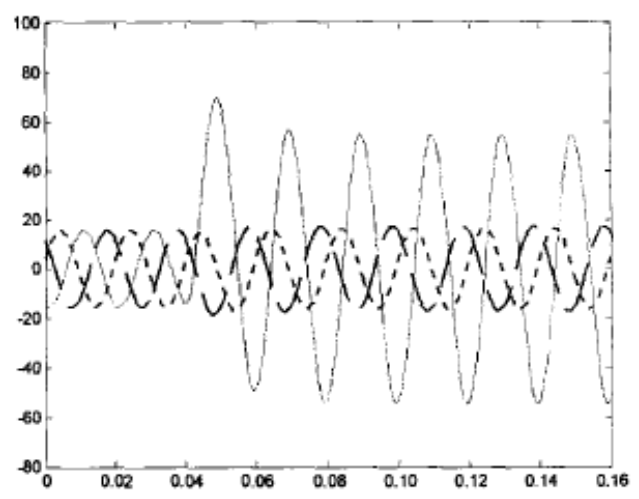

(b) neutral point with $16 \Omega$ reactance

Fig. 3 single phase fault current in $220 \mathrm{kV}$ side

Accurately grasping the impact on fault current of autotransformer neutral grounding via low reactance at the $500 \mathrm{kV}$ and $220 \mathrm{kV}$ side is necessary. Here, $0 \Omega$ (ungrounded) $5 \Omega, 8 \Omega, 12 \Omega, 16 \Omega$ and $19 \Omega$ were selected for small reactance respectively. Simulations in three common faults were processed, and the results are shown in Table 1. 
Tab. 1 effect of earth reactance to fault current in $220 \mathrm{kV}$ side

\begin{tabular}{|c|c|c|c|c|}
\hline \multirow{2}{*}{\multicolumn{2}{|c|}{ Reactance }} & \multicolumn{3}{c|}{ Fault Type } \\
\cline { 2 - 5 } \multicolumn{2}{|c|}{0} & Single & Three phase & Two phase \\
\cline { 2 - 5 } & Current (kA) & 46.58 & 44.21 & 43.87 \\
\hline \multirow{3}{*}{5} & Decrease percent (\%) & 0 & 0 & 0 \\
\cline { 2 - 5 } & Current (kA) & 40.37 & 44.21 & 41.41 \\
\hline \multirow{3}{*}{8} & Decrease percent (\%) & 13.3 & 0 & 5.6 \\
\cline { 2 - 5 } & Current (kA) & 39.66 & 44.21 & 40.23 \\
\hline \multirow{3}{*}{12} & Decrease percent (\%) & 14.9 & 0 & 8.3 \\
\cline { 2 - 5 } & Current (kA) & 38.82 & 44.21 & 39.03 \\
\hline \multirow{3}{*}{16} & Decrease percent (\%) & 16.7 & 0 & 11.0 \\
\cline { 2 - 5 } & Current (kA) & 37.89 & 44.21 & 38.14 \\
\hline \multirow{3}{*}{19} & Decrease percent (\%) & 18.7 & 0 & 13.1 \\
\cline { 2 - 5 } & Current (kA) & 37.32 & 44.21 & 37.59 \\
\cline { 2 - 5 } & Decrease percent (\%) & 19.9 & 0 & 14.3 \\
\hline
\end{tabular}

As can be seen from the above table, adding small reactance could decrease single and two phase fault current in $220 \mathrm{kV}$ side. It can be reduced by nearly $20 \%$ in maximum. The larger the added reactance is, the more obvious the effect would be. But it hardly affects the three phase fault current.

\section{Conclusions}

As can be seen from the above analysis and simulation, autotransformer is different from the general one. General transformer will increase the single fault current when its neutral point is grounded directly. Reactance of autotransformer is smaller, because its high and medium windings have couple relationship. When single and two phase fault happen in $220 \mathrm{kV}$ side, earth of neutral point could decrease fault current. And the effect would be more obvious with the increasing of earth reactance. Based on rules, autotransformer should be connected small reactance whose value is smaller than $U_{k h-m} \% / 3$. While, it has no effect to three phase fault current.

\section{References}

[1] Guven, A. N, Sebo, S.A. Analysis of ground fault current distribution along underground cables. IEEE Transactions on Power Delivery. 1986

[2] Zamora, I, Mazon, A.J.,Sagastabeitia, K.J. New method for detecting low current faults in electrical distribution systems. IEEE Transactions on Power Delivery. 2007

[3] Morrison, W.G,Arhart, R.J. Using new technology to improve reliability of an industrial cable distribution system. IEEE Transactions on Industrial Applications. 1992

[4] Owen,E. L. The historical development of neutral grounding Practices. IEEE Industry Applications Magazine. 1997

[5] Yao Huarmian, Cao Meiyue. The optimization on operation characteristics of MVnetwork. Power System Technology. 1998 